\title{
Harmful impact of profenofos on the physiological parameters in Nile tilapia, Oreochromis niloticus
}

\author{
Khaled Sharafeldin ${ }^{1}$, Hanan Abdel-Gawad ${ }^{2}$, Enas Ramzy ${ }^{2}$, Mohamed Sweilum ${ }^{3}$, Mohamed Nagy ${ }^{1}$ \\ ${ }^{1}$ Zoology Department, Faculty of Science, Benha University, Benha, Qaliobya, Egypt \\ ${ }^{2}$ National water Research, El-Kanater El-Kayria, Egypt \\ ${ }^{3}$ National Institute of Oceanography and Fisheries, Cairo, Egypt \\ *Corresponding author E-mail: keldeen@hotmail.com
}

Copyright (-) 2014 Khaled Sharafeldin et al. This is an open access article distributed under the Creative Commons Attribution License, which permits unrestricted use, distribution, and reproduction in any medium, provided the original work is properly cited.

\begin{abstract}
Profenofos as an organophosphorus insecticide has been used in the agricultural countries as Egypt, may find its way to water system and adversely effect on aquatic life particularly fish. Nile tilapia, Oreochromis niloticus as a major fish species in River Nile and one of the major sources of protein for human beings in Egypt, and it can also be a source of threaten to human health. Transport profenofos directly to tilapia fish may affect their physiological status and then fish production. The mortality of profenofos toxicity was estimated on tilapia and LC50 was detected as $0.87 \mathrm{mg} / \mathrm{l}$. Also fish were exposed to 1/2 LC50 for 96 hrs and to 1/10 LC50 for 28 days and lastly were left after the chronic toxicity for another 28 days as recovery period. The increase of blood glucose was accompanied with decrease in liver and muscles glycogen throughout the acute and chronic trail periods. Fish showed also highly significant decrease in serum total protein and globulin with increasing in albumin and $\mathrm{A} / \mathrm{G}$ ratio. A sharp elevation in serum creatinine, urea and uric acid with decrease in serum total lipid, triglyceride and cholesterol were also recorded. Lastly gradual and sharp elevation in the levels of serum enzymes, S-AST, S-ALT and S-ALP was revealed in profenofos-exposed tilapia. Same behavior as S-AST and S-ALT were in liver transaminases (L-AST \& L-ALT). Our study revealed adverse change of metabolism in tilapia due to profenofos exposure. This may inform about the dangerous use of profenofos and limitations should be managed.
\end{abstract}

\section{Introduction}

Egypt which is mainly an agricultural country has relied heavily on pesticides to control harmful pests mainly to cotton, maize, rice, wheat, bean and other cultivations [1]. The monitoring of pesticides residue in River Nile confirmed that natural fresh water are the ultimate recipients of most toxic substances generated by industrial, agricultural land run-off, drainage water and domestic activities [2]. Pesticides of interest here is the S-alkyl organophosphorus insecticide profenofos (selecron) which is moderately severe toxicity [3]. Profenofos has low persistence and are readily decomposed so it was used extensively during those years for selective control of mites on cotton, maize and other vegetables in Egypt. Profenofos like other pesticides may find its way to water system and adversely effect on aquatic life [4]. Consequently, several major fish death has occurred in recent years which have been traced to pesticides use. Nile tilapia, Oreochromis niloticus as a major fish species in River Nile, well marketable and are one of the major sources of protein for human beings in Egypt, and it can also be a source of threaten to human health by transporting the toxic material directly to consumers due to the bioaccumulation of pesticides in its flesh to a level that affects their physiological status and then fish production [5].

The aim of this study were to show the effects of acute and chronic toxicity of organophosphorus pesticide (profenofos) on biochemical analysis and enzymatic activities of serum and liver of Nile tilapia, O. niloticus. 


\section{Methods}

\subsection{Pesticide}

The pesticide has a common name is Profenofos (72\%) and commercial name is Selecron.

\subsubsection{Formula}

\section{C11H15 BrClO3PS}

\subsubsection{Chemical name}

O-(4-bromo-2-chlorophenyl) O-ethyl S-propylphosphorothioate

Profenofos has a molecular weight 373.6, Density $1.455\left(20^{\circ} \mathrm{C}\right)$, Solubility $28 \mathrm{mg} / \mathrm{l}\left(25^{\circ} \mathrm{C}\right)$, Hydrolysis 14.6 days (pH7) and Toxicity of Class (II) (MANIUAL PESTICIDE). Insecticide, acaricide profenofos is an organophosphorus insecticide developed for selective control of mites on cotton, maize, sugar beet, Soya beans, tomatoes, potatoes, vegetables, tobacco and palms. Insecticide introduced by Syngenta Agro-Pazel Switzerland (C.T.C.).

\subsection{Fish}

Nile tilapia, Oreochromis niloticus used in present study have average total length $16-18.3 \mathrm{~cm}$ and average total weight 80-100g. The fish were collected from Islamic Company for Animal Productions, Alkanater, Egypt and transported to the laboratory in plastic ice-box containing dechlorinated-oxygenated water. Fish were acclimatized for three weeks in aerated fiber glass tanks before use. The physico-chemical characteristics of the test water were measured and recorded as $\mathrm{pH} 7.9$, temperature $25^{\circ} \mathrm{C}$ and hardness $194.03 \pm 1.83 \mathrm{mg} / \mathrm{l}$ as $\mathrm{CaCO}$. Fish were fed on a commercial diet $(30 \%$ protein). Aquariums were changed once daily to remove any feces and uneaten food from the previous day. Fish were transferred randomly into experimental glass aquaria and were acclimatized for one week before starting the exposure to tested insecticide.

\subsection{Determination of profenofos LC50}

Nine concentrations $(0.7,0.8,0.85,0.87,0.9,1,3,4$ and $5 \mathrm{mg} / \mathrm{l})$ of profenofos were prepared in nine equal-sized aquaria, in addition to one aquarium for control. Ten fishes were transferred to individual profenofos containing aquaria. The experiment was continued for $96 \mathrm{hrs}$ of each concentration. Water was changed daily to keep profenofos concentration constant during 96 hrs. The fish died were watched and removed per day. By the end of the fourth day, the mortality percentages were then calculated according to probit analysis method [6]. This experiment was repeated twice and the average of mortality was taken. The LC50 value for profenofos was recorded as $0.87 \mathrm{mg} / \mathrm{l}$.

\subsection{Acute experiment for $96 \mathrm{hrs}$ to $1 / 2 \mathrm{LC50}$ of profenofos}

Fifty fish were transferred to individual aquaria contacting $1 / 2$ LC50 of profenofos $(0.435 \mathrm{mg} / \mathrm{l})$ for 96 hrs, in addition to control one. Then ten fish were sacrificed, $24 \mathrm{hrs}$ interval, during the acute exposure period, in addition to 10 fish for control group.

\subsection{Chronic experiment for 28 days to $1 / 10$ LC50 of profenofos}

Ninety fish were transferred to nine individual aquaria (10 fish each) containing 1/10 LC50 of profenofos (0.087 mg/l) for $1,2,3,4,7,14,21$ and 28 days, in addition to ninety fish as control one. Then ten fish were sacrificed at previously mentioned intervals of profenofos, during the exposure period, in addition to 10 fish from the control or non-profenofos aquaria.

\subsection{Recovery group experiment}

The rest of 10 fish exposed to 1/10 LC50 of profenofos $(0.087 \mathrm{mg} / \mathrm{l})$ for 28 days were transferred to dechlorinated and oxygenated profenofos free water for another 28 days, as one interval, then sacrificed.

\subsection{Sample}

Blood, liver and muscle tissues were obtained from all sacrificed fish in the current study. Sera were obtained and kept with liver and muscle tissues at $-20^{\circ} \mathrm{C}$ for biochemical analysis and enzyme activity measurement. 


\subsection{Serum and liver metabolites}

Serum glucose was determined according to Trinder [7] which based on GOD-PAP method (enzymatic colorimetric test). Liver and muscle glycogen determined by using the anthrone method according to Trevelyan and Harrison [8]. Total protein was determined based on Biuret method [9]. Determination of serum albumin was done according to bromocresol green method which described by Doumas [10]. Serum globulin can be calculated by subtracting the amount of serum albumin from the serum total protein. A/G ratio was calculated by the equation Albumin/globulin x100. Creatinine was measured by using Kinetic JAFFE method according to Tatzidis [11]. Urea was determined by urease Berthelot reaction according to Patton \& Crouch [12]. Uric acid was determined by Uricase-PAP method (enzymatic colorimetric test) according to Fossati et al. [13]. Total lipid in fish serum was estimated calorimetrically by sulfposphovanillic mixture according to Zollner \& Kirsch [14]. Estimation of triglyceride was done by GPO-PAP method according to Fossati \& Principe [15]. Serum cholesterol was determined as CHOD-PAP method according to Allain et al. [16].

\subsection{Serum and liver enzymes}

Serum aspartate aminotransferase (AST-E.C.2-6-1-1) and Alanine aminotransferase (ALT-E-C-2-6-1.2) activities were estimated by enzymatic colorimetric test according to Schmidt \& Schmidt [17]. Determination of alkaline phosphatase (ALP-E.C.3-1-3-1) based on phenyl phosphate colorimetric method as described by Belfield \& Goldberg [18]. For determination of liver enzymes $0.5 \mathrm{~g}$ of liver was taken and homogenized in $4 \mathrm{ml}$ cooled distilled water. Then the sample was centrifuged at 5000 r.p.m for 15 minutes. The supernatant was kept in dry test tube for determination of the activities of both transaminases.

\subsection{Statistical analysis}

The statistical analysis was estimated according to the method of Snedecor \& Cochran [19]. Results were expressed as mean \pm SE. The intergroup variation was measured by one way analysis of variance (ANOVA) followed by Tukey's LSD test.

\section{Results}

\subsection{Acute exposure}

\subsubsection{Serum and liver metabolites}

Exposure of Nile tilapia, Oreochromis niloticus to $0.435 \mathrm{mg}$ profenofos/1 (1/2 LC50) for 96 hrs showed sharp significant increase $(\mathrm{P}<0.05)$ of blood glucose level where it reached its peak after 96 hrs (Table 1). The exposed tilapia revealed a highly significant decrease of liver and muscle glycogen $(\mathrm{P}<0.05)$ during all the exposure period, where it reaches less than the half the control mean value after $96 \mathrm{hrs}$ (Table 1). The profenofos-exposed fish showed highly significant decrease $(\mathrm{P}<0.05)$ of total protein and globulin $($ Table 1$)$. On the other hand, serum albumin of tilapia significantly increased $(\mathrm{P}<0.05), \mathrm{A} / \mathrm{G}$ ratio of tilapia exposed to the same concentration of profenofos significantly increased $(\mathrm{P}<0.05)$ (Table 1). As serum creatinine, urea and uric acid levels were significantly increased $(\mathrm{P}<0.05)$ when fish were exposed to $0.435 \mathrm{mg}$ profenofos/L for $96 \mathrm{hrs}$ (Table 2). Simultaneously, serum total lipid, triglyceride and cholesterol levels were decreased significantly in profenofos-exposed fish $(\mathrm{P}<0.05)$ (Table 2).

\subsubsection{Serum and liver enzymes}

Highly elevated levels of serum AST, ALT and ALP were recorded when fish exposed acutely to profenofos (P<0.05) (Table 3). Liver enzymes, AST and ALT also recorded increased levels in fish of the same exposure $(\mathrm{P}<0.05)($ Table 3$)$.

\subsection{Chronic exposure}

\subsubsection{Serum and liver metabolites}

It was observed that whereas levels of glucose increased proportionally with time of chronic exposure, liver and muscle glycogen decreased dramatically $(\mathrm{P}<0.05)$ (Table 4). Levels of serum total protein were decreased during the first two weeks $(\mathrm{P}<0.05)$ while the effect was reversed to significant increase during the last two weeks $(\mathrm{P}<0.05)($ Table 4$)$. Serum albumin levels were significantly increased gradually $(\mathrm{P}<0.05)$ by increasing the exposure period to fish, while serum globulin showed the opposite behavior proportionally with the exposure time (Table 4). Concerning the A/G 
ratio, gradual significant elevation $(\mathrm{P}<0.05)$ was cleared where it reached its peak within 7 days $($ Table 4$)$. Serum creatinine showed highly significant increase $(\mathrm{P}<0.05)$ after 7 days till the end of chronic period (Table 5). Similarly, serum urea and uric acid were started to increase significantly after 4 days of exposure till the end of profenofos exposure period (Table 5). The present data indicated a gradual decrease in total lipid during the first 7 days $(\mathrm{P}<0.05)$ of profenofos exposure (Table 5). The effect was reversed with a highly significant elevation $(\mathrm{P}<0.05)$ after 14 days, 21 days and 28 days of exposure (Table 5). The same effect as total lipid was seen in triglyceride and cholesterol but the reversed data started after 7 days (Table 5).

Table 1: Changes of Glucose, Liver Glycogen, Muscle Glycogen, Total Protein, Albumin, Globulin and A/G Ratio of Nile Tilapia after Acute Exposure to $0.435 \mathrm{Mg}$ Profenofos/L.

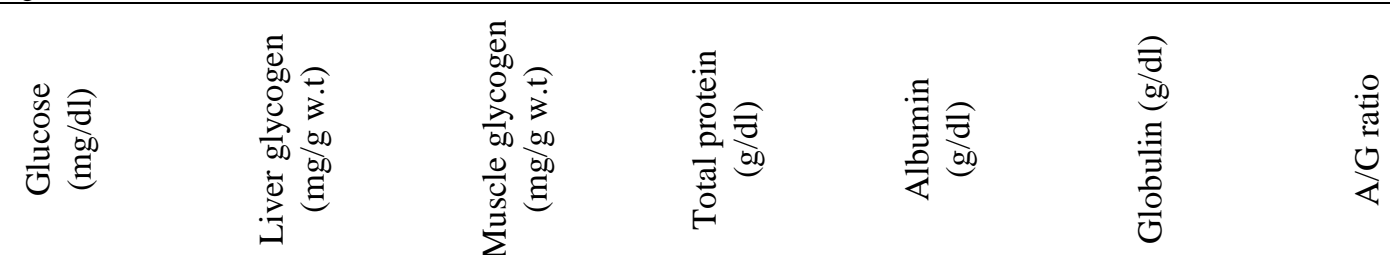

\begin{tabular}{llllllll}
\hline Control & $68.3 \pm 0.8^{\mathrm{e}}$ & $13.4 \pm 0.06^{\mathrm{a}}$ & $7.1 \pm 0.06^{\mathrm{a}}$ & $6.6 \pm 0.04^{\mathrm{a}}$ & $1.5 \pm 0.1^{\mathrm{c}}$ & $5.1 \pm 0.1^{\mathrm{a}}$ & $30.0 \pm 0.2^{\mathrm{d}}$ \\
24hrs. & $92.9 \pm 0.4^{\mathrm{d}}$ & $8.3 \pm 0.05^{\mathrm{b}}$ & $5.9 \pm 0.02^{\mathrm{b}}$ & $5.1 \pm 0.04^{\mathrm{b}}$ & $2.1 \pm 0.02^{\mathrm{b}}$ & $3.0 \pm 0.3^{\mathrm{b}}$ & $72.5 \pm 0.1^{\mathrm{cd}}$ \\
$48 \mathrm{hrs}$. & $102.5 \pm 0.8^{\mathrm{c}}$ & $7.5 \pm 0.04^{\mathrm{c}}$ & $5.2 \pm 0.01^{\mathrm{c}}$ & $4.7 \pm 0.03^{\mathrm{c}}$ & $2.2 \pm 0.03^{\mathrm{ab}}$ & $2.4 \pm 0.08^{\mathrm{c}}$ & $94.5 \pm 0.3^{\mathrm{bc}}$ \\
$72 \mathrm{hrs}$. & $113.1 \pm 1.0^{\mathrm{b}}$ & $6.8 \pm 0.04^{\mathrm{d}}$ & $4.8 \pm 0.03^{\mathrm{d}}$ & $3.9 \pm 0.03^{\mathrm{d}}$ & $2.3 \pm 0.01^{\mathrm{a}}$ & $1.6 \pm 0.07^{\mathrm{d}}$ & $145.2 \pm 0.1^{\mathrm{b}}$ \\
$96 \mathrm{hrs}$. & $129.9 \pm 0.7^{\mathrm{a}}$ & $5.6 \pm 0.06^{\mathrm{d}}$ & $3.9 \pm 0.03^{\mathrm{e}}$ & $3.6 \pm 0.03^{\mathrm{d}}$ & $2.3 \pm 0.02^{\mathrm{a}}$ & $1.2 \pm 0.08^{\mathrm{d}}$ & $231.5 \pm 0.3^{\mathrm{a}}$ \\
\hline
\end{tabular}

All data are mean of 10 individuals. Data are expressed as mean \pm SE. Variation between similar single letters in each component is not significant.

Table 2: Changes of Creatinine, Urea, Uric Acid, Total Lipid, Triglyceride and Cholesterol of Nile Tilapia after Acute Exposure to 0.435 Mg Profenofos/L.

\begin{tabular}{|c|c|c|c|c|c|c|}
\hline & 导 & 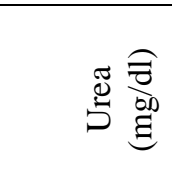 & 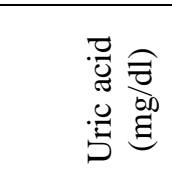 & 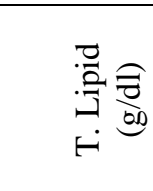 & 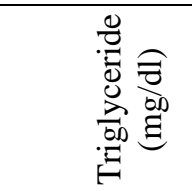 & 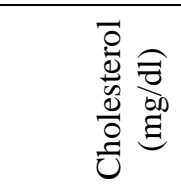 \\
\hline Control & $1.03 \pm 0.08^{\mathrm{e}}$ & $8.5 \pm 0.24^{\mathrm{e}}$ & $6.1 \pm 0.09^{d}$ & $12.8 \pm 0.1^{\mathrm{a}}$ & $258.6 \pm 1.2^{\mathrm{a}}$ & $162.1 \pm 0.5^{\mathrm{a}}$ \\
\hline 24hrs. & $1.2 \pm 0.07^{\mathrm{d}}$ & $12.2 \pm 0.09^{\mathrm{d}}$ & $8.5 \pm 0.03^{\mathrm{c}}$ & $9.7 \pm 0.08^{b}$ & $194.8 \pm 0.3^{b}$ & $137.1 \pm 0.43^{b}$ \\
\hline 48hrs. & $1.9 \pm 0.02^{\mathrm{c}}$ & $16.1 \pm 0.04^{\mathrm{c}}$ & $9.1 \pm 0.13^{c}$ & $9.0 \pm 0.06^{b}$ & $167.3 \pm 0.46^{\mathrm{c}}$ & $127.4 \pm 0.6^{\mathrm{c}}$ \\
\hline $72 \mathrm{hrs}$. & $2.2 \pm 0.01^{\mathrm{b}}$ & $17.6 \pm 0.1^{\mathrm{b}}$ & $11.1 \pm 0.07^{\mathrm{b}}$ & $7.4 \pm 0.1^{\mathrm{c}}$ & $139.3 \pm 0.8^{\mathrm{d}}$ & $115.2 \pm 0.74^{\mathrm{d}}$ \\
\hline 96hrs. & $2.8 \pm 0.04^{\mathrm{a}}$ & $20.6 \pm 0.2^{a}$ & $14.5 \pm 0.1^{\mathrm{a}}$ & $6.7 \pm 0.06^{\mathrm{c}}$ & $115.3 \pm 0.6^{\mathrm{e}}$ & $98.1 \pm 0.8^{\mathrm{d}}$ \\
\hline
\end{tabular}

All data are mean of 10 individuals. Data are expressed as mean \pm SE. Variation between similar single letters in each component is not significant.

Table 3: Changes of Serum and Liver Enzyme Activities; AST, ALT and ALK of Nile Tilapia after Acute Exposure to 0.435 Mg Profenofos/L

\begin{tabular}{llllll}
\hline & S. AST & S. ALT & S. ALP & L. AST & L. ALT \\
\hline Control & $25.7 \pm 0.3^{\mathrm{e}}$ & $17.9 \pm 0.3^{\mathrm{e}}$ & $13.34 \pm 0.3^{\mathrm{d}}$ & $40.88 \pm 0.5^{\mathrm{e}}$ & $18.05 \pm 0.1^{\mathrm{d}}$ \\
$24 \mathrm{hrs.}$ & $56.7 \pm 0.4^{\mathrm{d}}$ & $30.8 \pm 0.2^{\mathrm{d}}$ & $28.73 \pm 0.5^{\mathrm{c}}$ & $81.31 \pm 0.9^{\mathrm{d}}$ & $22.87 \pm 0.3^{\mathrm{c}}$ \\
$48 \mathrm{hrs}$. & $65.4 \pm 0.9^{\mathrm{c}}$ & $42.1 \pm 0.6^{\mathrm{b}}$ & $31.92 \pm 0.6^{\mathrm{c}}$ & $111.54 \pm 0.1^{\mathrm{c}}$ & $27.17 \pm 0.4^{\mathrm{c}}$ \\
$72 \mathrm{hrs}$. & $92.2 \pm 0.9^{\mathrm{d}}$ & $63.7 \pm 1.1^{\mathrm{b}}$ & $40.82 \pm 0.9^{\mathrm{b}}$ & $140.03 \pm 1.04^{\mathrm{b}}$ & $49.24 \pm 0.8^{\mathrm{b}}$ \\
$96 \mathrm{hrs}$. & $110.7 \pm 1.1^{\mathrm{a}}$ & $78.6 \pm 0.9^{\mathrm{a}}$ & $50.11 \pm 0.6^{\mathrm{a}}$ & $163.39 \pm 1.01^{\mathrm{a}}$ & $67.5 \pm 0.6^{\mathrm{a}}$ \\
\hline
\end{tabular}

All data are mean of 10 individuals. Data are expressed as mean \pm SE. Variation between similar single letters in each component is not significant.

\subsubsection{Serum and liver enzymes}

Serum AST, ALT and ALP enzymes recorded gradual and significant elevation of activities during the whole exposure period in acute and chronic experiments (Table $3 \& 6$ ). On the other hand only ALT and ALP were returned to the control values but AST levels were kept higher (Table 6). Parallel to sera enzymes, activities of liver AST and ALT enzymes revealed gradual increase during the exposure period $(\mathrm{P}<0.05)$ (Table 6). Liver ALT cleared no significant differences at 1, 2 and 7 days in comparison with corresponding controls, beside a sudden drop after 7 days when compared to the other exposed fish (Table 6). The enzymes' activity decreased significantly $(\mathrm{P}<0.05)$ after recovery but still higher than control values (Table 6). 
Table 4: Changes of Glucose,Liver Glycogen, Muscle Glycogen, Total Protein, Albumin, Globulin and A/G Ratio of Nile Tilapia after Acute Exposure to $0.087 \mathrm{Mg}$ Profenofos/L.

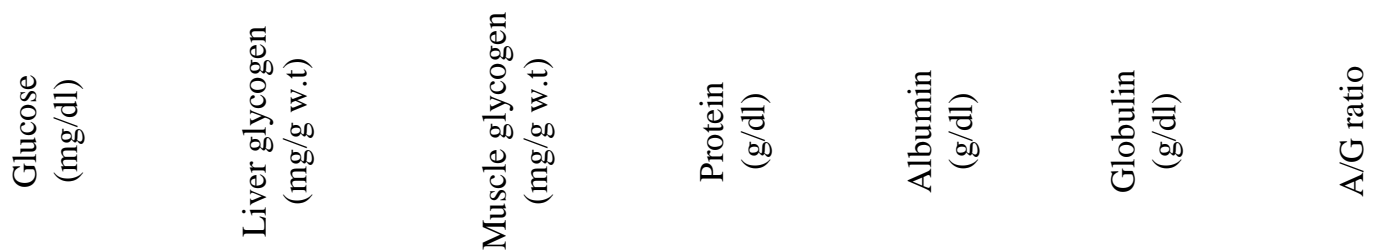

\begin{tabular}{|c|c|c|c|c|c|c|c|}
\hline Control & $68.3 \pm 0.8^{f}$ & $13.4 \pm 0.1^{\mathrm{a}}$ & $7.1 \pm 0.06^{\mathrm{a}}$ & $6.6 \pm 0.04^{b c}$ & $1.5 \pm 0.01^{\mathrm{ef}}$ & $5.1 \pm 0.3^{\mathrm{abc}}$ & $30.1 \pm 0.5^{\text {ghi }}$ \\
\hline 1 day & $73.0 \pm 0.7^{\mathrm{f}}$ & $12.0 \pm 0.04^{\mathrm{d}}$ & $6.9 \pm 0.01^{\mathrm{ab}}$ & $6.3 \pm 0.01^{\mathrm{d}}$ & $1.7 \pm 0.01^{\mathrm{d}}$ & $4.6 \pm 0.2^{\mathrm{de}}$ & $36.5 \pm 0.5^{\mathrm{ef}}$ \\
\hline Control & $68.3 \pm 0.8^{\mathrm{fg}}$ & $13.4 \pm 0.06^{\mathrm{a}}$ & $7.1 \pm 0.06^{\mathrm{a}}$ & $6.6 \pm 0.04^{\mathrm{bc}}$ & $1.5 \pm 0.01^{\mathrm{ef}}$ & $5.1 \pm 0.3^{\mathrm{abc}}$ & $30.1 \pm 0.5^{\text {ghi }}$ \\
\hline 2 days & $78.5 \pm 0.6^{\mathrm{e}}$ & $10.6 \pm 0.04^{\mathrm{e}}$ & $6.5 \pm 0.03^{\mathrm{c}}$ & $6.1 \pm 0.01^{\mathrm{d}}$ & $1.9 \pm 0.01^{\mathrm{c}}$ & $4.2 \pm 0.2^{\mathrm{e}}$ & $43.9 \pm 0.45^{\mathrm{d}}$ \\
\hline Control & $68.3 \pm 0.8^{\mathrm{fg}}$ & $13.4 \pm 0.06^{\mathrm{a}}$ & $7.1 \pm 0.06^{\mathrm{a}}$ & $6.6 \pm 0.04^{b c}$ & $1.5 \pm 0.01^{\mathrm{ef}}$ & $5.1 \pm 0.3^{\mathrm{abc}}$ & $30.1 \pm 0.5^{\text {ghi }}$ \\
\hline 3 days & $87.6 \pm 0.8^{\mathrm{d}}$ & $10.2 \pm 0.04^{\mathrm{f}}$ & $6.2 \pm 0.02^{\mathrm{d}}$ & $5.5 \pm 0.03^{\mathrm{e}}$ & $2.0 \pm 0.01^{\mathrm{ab}}$ & $3.5 \pm 0.4^{\mathrm{f}}$ & $57.6 \pm 0.63^{c}$ \\
\hline Control & $68.3 \pm 0.8^{\mathrm{fg}}$ & $13.4 \pm 0.06^{\mathrm{a}}$ & $7.1 \pm 0.06^{\mathrm{a}}$ & $6.6 \pm 0.04^{b c}$ & $1.5 \pm 0.01^{\mathrm{ef}}$ & $5.1 \pm 0.3^{\mathrm{abc}}$ & $30.1 \pm 0.5^{\text {ghi }}$ \\
\hline 4 days & $89.1 \pm 0.2^{\mathrm{d}}$ & $9.781 \pm 0.033^{g}$ & $5.9 \pm 0.02^{\mathrm{d}}$ & $5.1 \pm 0.02^{\mathrm{f}}$ & $2.0 \pm 0.01^{\mathrm{abc}}$ & $3.1 \pm 0.5^{\mathrm{g}}$ & $62.9 \pm 0.7^{b}$ \\
\hline Control & $66.1 \pm 0.8^{\mathrm{gh}}$ & $13.07 \pm 0.04^{\mathrm{ab}}$ & $6.9 \pm 0.05^{\mathrm{ab}}$ & $6.8 \pm 0.04^{\mathrm{bc}}$ & $1.4 \pm 0.01^{\mathrm{f}}$ & $5.3 \pm 0.9^{\mathrm{ab}}$ & $27.5 \pm 0.5^{\mathrm{hi}}$ \\
\hline 7 days & $94.0 \pm 0.5^{\mathrm{c}}$ & $8.76 \pm 0.06^{\mathrm{h}}$ & $5.6 \pm 0.02^{\mathrm{e}}$ & $4.3 \pm 0.02^{\mathrm{g}}$ & $1.9 \pm 0.01^{\mathrm{bc}}$ & $2.4 \pm 0.5^{\mathrm{h}}$ & $75.8 \pm 0.7^{\mathrm{a}}$ \\
\hline Control & $63.1 \pm 0.5^{\mathrm{gh}}$ & $12.8 \pm 0.03^{\mathrm{bc}}$ & $6.9 \pm 0.05^{\mathrm{ab}}$ & $6.7 \pm 0.06^{\mathrm{bc}}$ & $1.6 \pm 0.02^{\mathrm{de}}$ & $5.2 \pm 1.3^{\mathrm{abc}}$ & $31.4 \pm 0.5^{\mathrm{gh}}$ \\
\hline 14 days & $98.7 \pm 0.2^{\mathrm{bc}}$ & $8.2 \pm 0.05^{\mathrm{i}}$ & $5.2 \pm 0.02^{\mathrm{f}}$ & $5.7 \pm 0.02^{\mathrm{e}}$ & $2.0 \pm 0.06^{\mathrm{ab}}$ & $3.7 \pm 5.2^{\mathrm{f}}$ & $53.8 \pm 0.83^{\mathrm{c}}$ \\
\hline Control & $63.5 \pm 0.6^{\mathrm{gh}}$ & $13.27 \pm 0.053^{\mathrm{a}}$ & $6.7 \pm 0.06^{\mathrm{bc}}$ & $6.5 \pm 0.06^{\mathrm{cd}}$ & $1.5 \pm 0.01^{\mathrm{ef}}$ & $4.9 \pm 1.7^{\mathrm{bc}}$ & $31.6 \pm 0.47^{\mathrm{gh}}$ \\
\hline 21 days & $100.4 \pm 0.2^{b}$ & $7.91 \pm 0.06^{\mathrm{i}}$ & $4.9 \pm 0.02^{\mathrm{g}}$ & $6.9 \pm 0.04^{\mathrm{ab}}$ & $2.0 \pm 0.01^{\mathrm{a}}$ & $4.9 \pm 0.8^{\mathrm{cd}}$ & $41.4 \pm 0.6^{\mathrm{d}}$ \\
\hline Control & $61.4 \pm 0.3^{\mathrm{h}}$ & $13.0 \pm 0.03^{\mathrm{ab}}$ & $6.9 \pm 0.02^{\mathrm{ab}}$ & $6.9 \pm 0.04^{\mathrm{ab}}$ & $1.4 \pm 0.01^{\mathrm{f}}$ & $5.4 \pm 0.3^{\mathrm{a}}$ & $26.5 \pm 0.6^{\mathrm{i}}$ \\
\hline 28 days & $107.6 \pm 0.8^{\mathrm{a}}$ & $6.9 \pm 0.024^{j}$ & $4.0 \pm 0.03^{\mathrm{h}}$ & $7.2 \pm 0.04^{\mathrm{a}}$ & $2.1 \pm 0.01^{\mathrm{a}}$ & $5.1 \pm 4.9^{\mathrm{abc}}$ & $40.2 \pm 0.5^{\mathrm{de}}$ \\
\hline Control & $61.4 \pm 0.3^{\mathrm{h}}$ & $13.2 \pm 0.03^{\mathrm{ab}}$ & $6.8 \pm 0.026^{\mathrm{abc}}$ & $6.8 \pm 0.04^{\mathrm{bc}}$ & $1.6 \pm 0.02^{\mathrm{de}}$ & $5.1^{\mathrm{a}} \pm 0.3^{\mathrm{bc}}$ & $31.3 \pm 0.5^{\text {gh }}$ \\
\hline Recovery & $12.6 \pm 0.05^{\mathrm{c}}$ & $12.6 \pm 0.05^{\mathrm{c}}$ & $6.1 \pm 0.04^{\mathrm{d}}$ & $6.9 \pm 0.02^{\mathrm{ab}}$ & $1.7 \pm 0.01^{\mathrm{d}}$ & $5.2 \pm 1.9^{\mathrm{abc}}$ & $32.7 \pm 0.83^{\mathrm{fg}}$ \\
\hline
\end{tabular}

All data are mean of 10 individuals. Data are expressed as mean \pm SE. Variation between similar single letters in each component is not significant.

Table 5: Changes of Creatinine, Urea, Uric Acid, Total Lipid, Triglyceride and Cholesterol of Nile Tilapia after Acute Exposure to 0.087 Mg Profenofos/L.

\begin{tabular}{|c|c|c|c|c|c|c|}
\hline & 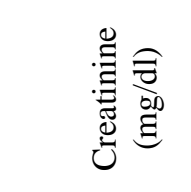 & 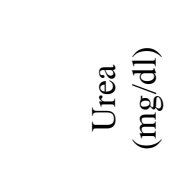 & 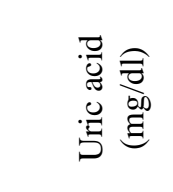 & 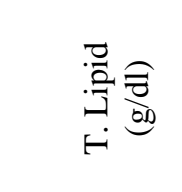 & 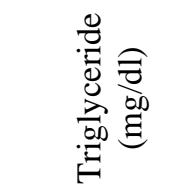 & 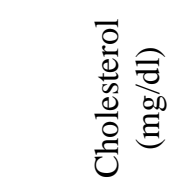 \\
\hline Control & $1.0 \pm 0.1^{\mathrm{fg}}$ & $8.5 \pm 0.024^{g h i}$ & $6.1 \pm 0.09^{g h}$ & $12.8 \pm 0.1^{\mathrm{c}}$ & $258.6 \pm 1.2^{\mathrm{c}}$ & $162.1 \pm 0.5^{\mathrm{c}}$ \\
\hline 1 day & $1.0 \pm 0.1^{\mathrm{fg}}$ & $9.4 \pm 0.08^{\text {fgh }}$ & $6.3 \pm 0.05^{\mathrm{gh}}$ & $11.5 \pm 0.07^{\mathrm{de}}$ & $226.6 \pm 0.6^{\mathrm{d}}$ & $137.0 \pm 0.5^{\mathrm{d}}$ \\
\hline Control & $1.0 \pm 0.1^{\mathrm{fg}}$ & $8.5 \pm 0.024^{\text {ghi }}$ & $6.1 \pm 0.09^{g h}$ & $12.8 \pm 0.1^{\mathrm{c}}$ & $258.6 \pm 1.2^{c}$ & $162.1 \pm 0.5^{\mathrm{c}}$ \\
\hline 2 days & $1.1 \pm 0.01^{\mathrm{efg}}$ & $9.6 \pm 0.09^{\mathrm{efg}}$ & $6.6 \pm 0.04^{\mathrm{fg}}$ & $11.3 \pm 0.07^{\mathrm{ef}}$ & $189.3 \pm 0.9^{\mathrm{e}}$ & $131.2 \pm 0.35^{\mathrm{de}}$ \\
\hline Control & $1.0 \pm 0.08^{\mathrm{fg}}$ & $8.5 \pm 0.02^{\text {ghi }}$ & $6.1 \pm 0.09^{g h}$ & $12.8 \pm 0.1^{\mathrm{c}}$ & $258.6 \pm 1.2^{c}$ & $162.1 \pm 0.5^{\mathrm{c}}$ \\
\hline 3 days & $1.1 \pm 0.01^{\mathrm{efg}}$ & $9.9 \pm 0.1^{\mathrm{ef}}$ & $7.2 \pm 0.04^{\mathrm{ef}}$ & $10.7 \pm 0.05^{\mathrm{f}}$ & $176.3 \pm 0.4^{\mathrm{f}}$ & $126.2 \pm 0.5^{\mathrm{e}}$ \\
\hline Control & $1.0 \pm 0.08^{\text {fg }}$ & $8.5 \pm 0.02^{\text {ghi }}$ & $6.1 \pm 0.09^{\mathrm{gh}}$ & $12.8 \pm 0.1^{\mathrm{c}}$ & $258.6 \pm 1.2^{c}$ & $162.1 \pm 0.5^{\mathrm{c}}$ \\
\hline 4 days & $1.2 \pm 0.02^{\mathrm{ef}}$ & $10.5 \pm 0.1^{\mathrm{de}}$ & $7.5 \pm 0.8^{\mathrm{e}}$ & $10.4 \pm 0.06^{\mathrm{f}}$ & $144.3 \pm 0.8^{\mathrm{g}}$ & $114.3 \pm 0.7^{\mathrm{f}}$ \\
\hline Control & $1.0 \pm 0.1^{\mathrm{fg}}$ & $7.9 \pm 0.08^{\mathrm{i}}$ & $5.9 \pm 0.08^{\mathrm{gh}}$ & $12.4 \pm 0.1^{\mathrm{cd}}$ & $257.8 \pm 0.5^{\mathrm{c}}$ & $162.0 \pm 0.5^{\mathrm{c}}$ \\
\hline 7 days & $1.3 \pm 0.01^{\mathrm{d}}$ & $11.1 \pm 0.13^{\mathrm{cd}}$ & $8.6 \pm 0.06^{\mathrm{d}}$ & $9.5 \pm 0.05^{\mathrm{g}}$ & $266.9 \pm 0.7^{b}$ & $173.9 \pm 0.9^{b}$ \\
\hline Control & $1.01 \pm 0.01^{\mathrm{fg}}$ & $8.2 \pm 0.09^{\mathrm{hi}}$ & $5.8 \pm 0.03^{g h}$ & $12.5 \pm 0.1^{\mathrm{cd}}$ & $253.3 \pm 0.8^{c}$ & $156.8 \pm 0.7^{\mathrm{c}}$ \\
\hline 14 days & $1.81 \pm 0.014^{\mathrm{c}}$ & $12.7 \pm 0.2^{c}$ & $9.4 \pm 0.1^{\mathrm{c}}$ & $13.9 \pm 0.07^{b}$ & $269.3 \pm 0.7^{b}$ & $176.7 \pm 1.0^{b}$ \\
\hline Control & $0.99 \pm 0.011^{\mathrm{fg}}$ & $7.5 \pm 0.09^{\mathrm{i}}$ & $5.6 \pm 0.06^{h}$ & $12.1 \pm 0.1^{\mathrm{cde}}$ & $253.0 \pm 0.6^{\mathrm{c}}$ & $159.5 \pm 0.7^{\mathrm{c}}$ \\
\hline 21 days & $2.04 \pm 0.02^{\mathrm{b}}$ & $15.6 \pm 0.1^{b}$ & $10.4 \pm 0.09^{b}$ & $16.2 \pm 0.06^{\mathrm{a}}$ & $270.2 \pm 0.8^{b}$ & $189.5 \pm 0.9^{a}$ \\
\hline Control & $0.95 \pm 0.013^{\mathrm{g}}$ & $7.4 \pm 0.1^{\mathrm{i}}$ & $5.5 \pm 0.06^{\mathrm{h}}$ & $11.6 \pm 0.1^{\mathrm{de}}$ & $251.9 \pm 0.8^{c}$ & $161.1 \pm 0.5^{\mathrm{c}}$ \\
\hline 28 days & $3.1 \pm 0.04^{\mathrm{a}}$ & $17.9 \pm 0.1^{\mathrm{a}}$ & $13.0 \pm 0.2^{\mathrm{a}}$ & $17.0 \pm 0.1^{\mathrm{a}}$ & $287.0 \pm 1.0^{\mathrm{a}}$ & $191.5 \pm 0.8^{a}$ \\
\hline Control & $1.01 \pm 1.01^{\mathrm{fg}}$ & $8.2 \pm 0.09^{\mathrm{hi}}$ & $5.3 \pm 0.06^{\mathrm{h}}$ & $11.6 \pm 0.1^{\mathrm{de}}$ & $253.5 \pm 0.8^{c}$ & $156.8 \pm 0.7^{\mathrm{c}}$ \\
\hline Recovery & $1.23 \pm 0.03^{\mathrm{de}}$ & $10.2 \pm 0.1^{\mathrm{def}}$ & $6.5 \pm 0.04^{\mathrm{fg}}$ & $12.4 \pm 0.1^{\mathrm{cd}}$ & $256.1 \pm 0.6^{\mathrm{c}}$ & $159.0 \pm 0.5^{\mathrm{c}}$ \\
\hline
\end{tabular}

All data are mean of 10 individuals. Data are expressed as mean \pm SE. Variation between similar single letters in each component is not significant. 
Table 6: Changes of Serum and Liver Enzyme Activities; AST, ALT and ALK of Nile Tilapia after Acute Exposure to 0.435 Mg Profenofos/L.

\begin{tabular}{llllll}
\hline & S. AST $(\mathrm{u} / 1)$ & S. ALT $(\mathrm{u} / \mathrm{l})$ & S. ALP $(\mathrm{u} / \mathrm{l})$ & L. AST $(\mathrm{u} / \mathrm{g})$ & L. ALT $(\mathrm{u} / \mathrm{g})$ \\
\hline Control & $25.7 \pm 0.3^{\mathrm{g}}$ & $17.9 \pm 0.3^{\mathrm{f}}$ & $13.3 \pm 0.3^{\mathrm{gh}}$ & $40.9 \pm 0.5^{\mathrm{h}}$ & $18.05 \pm 0.14^{\mathrm{f}}$ \\
1 day & $38.9 \pm 0.4^{\mathrm{f}}$ & $26.1 \pm 0.4^{\mathrm{e}}$ & $16.1 \pm 0.2^{\mathrm{fg}}$ & $43.9 \pm 0.5^{\mathrm{h}}$ & $18.6 \pm 0.1^{\mathrm{f}}$ \\
Control & $25.7 \pm 0.3^{\mathrm{g}}$ & $17.9 \pm 0.3^{\mathrm{f}}$ & $13.3 \pm 0.3^{\mathrm{gh}}$ & $40.9 \pm 0.5^{\mathrm{h}}$ & $18.05 \pm 0.14^{\mathrm{f}}$ \\
2 days & $42.0 \pm 0.5^{\mathrm{f}}$ & $32.0 \pm 0.5^{\mathrm{d}}$ & $19.0 \pm 0.2^{\mathrm{ef}}$ & $62.2 \pm 0.45^{\mathrm{f}}$ & $19.9 \pm 0.1^{\mathrm{f}}$ \\
Control & $25.7 \pm 0.3^{\mathrm{g}}$ & $17.9 \pm 0.3^{\mathrm{f}}$ & $13.3 \pm 0.3^{\mathrm{gh}}$ & $40.9 \pm 0.5^{\mathrm{h}}$ & $18.1 \pm 0.14^{\mathrm{f}}$ \\
3 days & $49.7 \pm 0.9^{\mathrm{e}}$ & $32.4 \pm 0.5^{\mathrm{d}}$ & $20.5 \pm 0.4^{\mathrm{e}}$ & $54.9 \pm 0.6^{\mathrm{g}}$ & $24.5 \pm 0.3^{\mathrm{e}}$ \\
Control & $25.7 \pm 0.3^{\mathrm{g}}$ & $17.9 \pm 0.3^{\mathrm{f}}$ & $13.3 \pm 0.3^{\mathrm{gh}}$ & $40.9 \pm 0.5^{\mathrm{h}}$ & $18.1 \pm 0.14^{\mathrm{f}}$ \\
4 days & $52.8 \pm 1.0^{\mathrm{e}}$ & $52.0 \pm 0.5^{\mathrm{c}}$ & $27.1 \pm 0.5^{\mathrm{d}}$ & $90.3 \pm 0.7^{\mathrm{d}}$ & $28.6 \pm 0.22^{\mathrm{d}}$ \\
Control & $26.1 \pm 3.2^{\mathrm{g}}$ & $16.3 \pm 3.3^{\mathrm{f}}$ & $11.9 \pm 0.9^{\mathrm{h}}$ & $43.7 \pm 0.5^{\mathrm{h}}$ & $18.5 \pm 0.2^{\mathrm{f}}$ \\
7 days & $69.6 \pm 9.6^{\mathrm{c}}$ & $63.9 \pm 8.9^{\mathrm{b}}$ & $35.9 \pm 0.5^{\mathrm{c}}$ & $90.3 \pm 0.7^{\mathrm{d}}$ & $20.1 \pm 0.15^{\mathrm{f}}$ \\
Control & $24.4 \pm 3.0^{\mathrm{g}}$ & $14.5 \pm 2.3^{\mathrm{f}}$ & $12.2 \pm 1.3^{\mathrm{gh}}$ & $39.7 \pm 0.5^{\mathrm{h}}$ & $20.0 \pm 0.2^{\mathrm{f}}$ \\
14 days & $87.0 \pm 9.9^{\mathrm{a}}$ & $76.7 \pm 7.6^{\mathrm{a}}$ & $39.8 \pm 5.2^{\mathrm{b}}$ & $118.4 \pm 0.8^{\mathrm{c}}$ & $33.3 \pm 0.3^{\mathrm{c}}$ \\
Control & $25.0 \pm 3.4^{\mathrm{g}}$ & $14.9 \pm 2.6^{\mathrm{f}}$ & $12.4 \pm 1.7^{\mathrm{gh}}$ & $39.2 \pm 0.47^{\mathrm{h}}$ & $18.9 \pm 0.1^{\mathrm{f}}$ \\
21 days & $80.8 \pm 6.0^{\mathrm{b}}$ & $51.9 \pm 12.2^{\mathrm{c}}$ & $43.6 \pm 0.8^{\mathrm{a}}$ & $131.5 \pm 0.6^{\mathrm{b}}$ & $36.0 \pm 0.3^{\mathrm{b}}$ \\
Control & $24.5 \pm 1.6^{\mathrm{g}}$ & $15.3 \pm 2.6^{\mathrm{f}}$ & $13.0 \pm 0.3^{\mathrm{gh}}$ & $41.01 \pm 0.6^{\mathrm{h}}$ & $18.5 \pm 0.1^{\mathrm{f}}$ \\
28 days & $62.6 \pm 8.6^{\mathrm{d}}$ & $48.9 \pm 11.3^{\mathrm{c}}$ & $33.1 \pm 4.9^{\mathrm{c}}$ & $141.5 \pm 0.5^{\mathrm{a}}$ & $41.7 \pm 0.4^{\mathrm{a}}$ \\
Control & $25.1 \pm 3.4^{\mathrm{g}}$ & $16.3 \pm 3.3^{\mathrm{f}}$ & $13.3 \pm 0.3^{\mathrm{gh}}$ & $40.9 \pm 0.5^{\mathrm{h}}$ & $19.9 \pm 0.2^{\mathrm{f}}$ \\
Recovery & $35.9 \pm 7.8^{\mathrm{f}}$ & $20.3 \pm 5.7^{\mathrm{f}}$ & $13.5 \pm 1.9^{\mathrm{gh}}$ & $87.1 \pm 0.8^{\mathrm{e}}$ & $26.7 \pm 0.3^{\mathrm{d}}$ \\
\hline
\end{tabular}

All data are mean of 10 individuals. Data are expressed as mean \pm SE. Variation between similar single letters in each component is not significant.

\section{Discussion}

The present study showed hyperglycemia after both acute and chronic exposure to profenofos in Nile tilapia. Accompanied to hyperglycemia a decrease of both liver and muscle glycogen content were recorded. This may be attributed to the blocking transfer of glucose into the cells which may lead to increased level of glucose in blood and decreased glycogenesis in liver and muscle cells. Same result has been mentioned after 24 weeks of exposure to organophosphate insecticides; malathion and dimethoate and after 28 days to deltamethrin on same fish species [20], [21]. They added that, hyperglycemia may be due to pesticide stress which leads to an increased secretion of catecholamines (glycogenolysis) and corticosteroid (gluconeogenesis) hormones. Jyothi \& Narayan [22] reported that, hyperglycemia might be attributed to partial or fully blocking process of glucose transport from blood stream to the liver by pesticide stress and impaired insulin secretion as result of pesticides toxicity. The exposure of Colisa fasciatus and O. niloticus to malathion and carbaryl, and deltamethrin, respectively showed same effects [20, 23]. Also, Matos et al. [24] revealed that such enhancement of glycogen breakdown in the liver of tilapia after carbaryl exposure is probably due to the toxicity effect of pesticides which caused hyperglycemia and increase of catecholamine secretion and corticosteroid hormones.

A decline in serum protein levels in Nile tilapia was also observed after acute exposure for 96 hrs to profenofos and chronically after 4 and 7 days of exposure. Hyperglycemia may impair the other liver function as protein synthesis or the pesticide stress may cause overuse of proteins. The other thing, liver cells may use much amount of amino acids to wave the shortage of non-transportable glucose molecules (gluconeogenesis activation). Similar results were also shown by Dutta et al. [25] after exposure of Indian catfish (Heteropneustes fossilis) to malathion, Kotb [1] after chronic exposure of O. niloticus to cinmethylin and anilofos and after acute exposure of Clarias gariepinus to both agricon and vertimic [26]. They could be attributed that decline to several pathological processes including plasma dissolution, renal damage and elimination in urine, decrease liver protein synthesis, alteration in hepatic blood flow and/or hemorrhage into peritoneal cavity and intestine. [26] added that direct enhancement use for muscle energy may lead to degradation of proteins and tissue breakdown as well as inhibition of protein synthesis. Unexpectedly the results were reversed and serum protein showed raised levels than control group after 21 and 28 days. This increase of protein may reflect the resistance or adaptation of fish to pesticide stress. Other study observed that reversed behavior. It attributed that response to increase in liver synthesis, disturbances in liver function or the immune response to pesticide which led to an increase in the formation of protein-insecticide complex [27]. Acute and chronic exposure of Nile tilapia to profenofos caused elevation of serum albumin and $\mathrm{A} / \mathrm{G}$ ratio, while decreased globulin level at all trial periods. Impairment of kidney functions which were estimated currently may cause albumin level imbalance and failure of stressed albumin excretion. Exposure of $\mathrm{C}$. carpio and $\mathrm{O}$.niloticus to dithiopyridine and paraquat showed same results [28], [29]. The increase in serum albumin was attributed to liver reaction, the hepatic cells began to synthesis more albumins that migrates to the blood for control and bound the pesticide toxic action [30], [31].

Acute and chronic exposure of Nile tilapia to profenofos also caused elevation in serum creatinine, urea and uric acid, these results were agree with many authors $[1,26,28]$. They revealed these elevations to the disturbances in the kidney and additionally due to necrosis of endothelial cells and renal hemopoietic tissue that was supported by Fischer-Scherl 
et al. [32]. In our results the increment levels of uric acid have started after 7 days of chronic exposure while creatinine and urea have started after 21 days, 2 weeks later, that may attributes the role of uric acid to raise the levels of creatinine and urea as kidney dysfunction indicators.

The present results showed decrease of total lipid content in the fish blood by the acute exposure for 96 hrs and chronically for 7 days. The reduction in total lipid was explained as a direct effect of the utilization of body fat as energy supply to meet the increasing physiological demands [33]. However Fayed et al. [34] attributed the serum total lipid reduction in fish to increase secretion of catecholamines as a result of pollutant stress which enhanced metabolic rate and in turn reduced metabolic reserves. This may be supported by the current depression of total protein and glycogen content in muscle and liver. The depression of triglyceride was in agree with Mekkawy et al. [35] after exposure of O. niloticus and C. auratus to atrazine for 28 days. The decline effect of total lipid was reversed after 14,21 and 28 days. Such reversal effect was also shown in triglyceride and cholesterol levels but has started as one week earlier, after 7 days of exposure. This figure may give impression that the change of triglyceride and cholesterol may be major cause of changing total lipid content. On the other hand, later increase of serum total lipids and triglycerides may be a result of partial or full inactivation of insulin as responses to pesticide toxicity in blood and the pancreatic tissues [36]. It promotes fat storage by activation of lipoprotein lipase and supplying breakdown product of glucose, $\alpha$ glycerophosphate which provides the glycerol portion of newly forming triglycerides [36]. Such irregular changes in the total lipid content were in agree with Kotb [1] during the exposure of O.niloticus to anilofos and cinmethylin for long term. Also, El-Amin [28] suggested the same result after both acute and chronic exposure of Cyprinus carpio to dithiopyridine. The depression of cholesterol was recorded in other fish by the exposure to diazinon, carbamate and phorate [33], [37]. They referred that reduction of cholesterol to the liver dysfunction which has an important role in cholesterol metabolism.

Regarding liver function, a highly significant increase of serum amino transferases; AST and ALT, and serum ALP were measured after both acute and chronic exposure to profenofos. Same results were also observed by El-Amin [28] and Fouda \& Azab [38] after exposures of C.carpio to dithiopyridine and Clarias gariepinus to vertemic and agricon. The increase in the activity of serum enzymes was explained as result of destruction of liver cells and increased cell permeability leading to a leakage of the enzymes from the damaged liver cells into serum [39]. Parallel to serum enzymes, both liver transferases levels were elevated as well. This increment of enzymes may be an indicator of liver damage. Same observations were found after chronic exposure of Tilapia mossambica to hepatachlor and Cyprinus carpio to ametryne [40], [41]. However authors referred that increase to liver damage, while alteration AST and ALT activity was correlated with the reduction in protein content in exposed fish.

The study of fish exposure to pesticide profenofos revealed an adverse effect on the metabolic processes and health of tilapia fish as an economic protein source.

\section{Disclosures}

The authors have no commercial associations that might be a conflict of interest in relation to this article.

\section{References}

[1] Kotb, G.A., Biochemical studies and remediation of Qarun lake Ph.D. Thesis. Fac. of Agric. Zagazig Univ., Egypt., 2007.

[2] Zidan, Z.H., et al., Survey of pesticides residue contaminating Nile water and agriculture eco-system at Kalubia govwernorate. Egypt. 1st Conf. of the Central. Agric. Pesticide Lab. , 2002: p. 139-155.

[3] Eddleston, M., et al., Poisoning with the S-Alkyl organophosphorus insecticides profenofos and prothiofos. QJM, $2009.102(11)$ : p. 785-92. http://dx.doi.org/10.1093/qjmed/hcp119.

[4] Ayas, Z., N. Barlas, and D. Kolankaya, Determination of organochlorine pesticide residues in various environments and organisms in Göksu Delta, Turkey. Aquatic Toxicology, 1997. 39(2): p. 171-181. http://dx.doi.org/10.1016/S0166-445X(96)00849-1.

[5] Zaghloul, K.H., Effect of different water sources on some biological and biochemical aspects of the Nile tilapia, Oreochromis niloticus and the Nile catfish, Claries gariepinus. . Egypt. J. Zool., 2000. 34 p. 353-377.

[6] Finney, D.J., Probit Analysis, 3rd ed. By D. J. Finney, Cambridge University Press, 32 E. 57th St., New York, Ny 10022, 1971. xv + 333 pp. 14.5 $\times 22 \mathrm{~cm}$. Price \$18.50. Journal of Pharmaceutical Sciences, 1971. 60(9): p. 1432-1432.

[7] Trinder, P., Enzymatic colorimetric method of glucose. . Ann. Clin. Biochem., 1969. 6.

[8] Trevelyan, W.E. and J.S. Harrison, Studies on yeast metabolism. I. Fractionation and microdetermination of cell carbohydrates. Biochem J, 1952. 50(3): p. 298-303.

[9] Gornall, A.G., C.J. Bardawill, and M.M. David, Determination of serum proteins by means of the biuret reaction. J Biol Chem, 1949. 177(2): p. 751-66.

[10]Doumas, B.T., W.A. Watson, and H.G. Biggs, Albumin standards and the measurement of serum albumin with bromcresol green. Clin Chim Acta, 1971. 31(1): p. 87-96. http://dx.doi.org/10.1016/0009-8981(71)90365-2.

[11] Yatzidis, H., New method for direct determination of "true" creatinine. Clin Chem, 1974. 20(9): p. 1131-4.

[12]Patton, C.J. and S.R. Crouch, Enzymatic colorimetric method of urea Anal. Chem. , $1977 . \quad$, 49 : p. 464 - 469. http://dx.doi.org/10.1021/ac50011a034.

[13] Fossati, P., L. Prencipe, and G. Berti, Use of 3,5-dichloro-2-hydroxybenzenesulfonic acid/4-aminophenazone chromogenic system in direct enzymic assay of uric acid in serum and urine. Clin Chem, 1980. 26(2): p. 227-31.

[14]Zollner, N. and K. Kirsch, Determination of lipids (micromethod) by means of the sulfophosphovanillin reaction common to many natural lipids. Z. Ges. Exp. Med., 1962. 135: p. 545 - 561. 
[15]Fossati, P. and L. Prencipe, Serum triglycerides determined colorimetrically with an enzyme that produces hydrogen peroxide. Clin Chem, 1982. 28(10): p. 2077-80.

[16] Allain, C.C., et al., Enzymatic determination of total serum cholesterol. Clin Chem, 1974. 20(4): p. 470-5.

[17]Schmidt, E. and F.W. Schmidt, Colorimetric methods of AST and ALT determination. . Enzym. Biol. Clin., 1963. 3.

[18]Belfield A and Goldberg DM, Colorimetric determination of alkaline phosphatase activity. Enzyme 1971. 12: p. 561.

[19] Snedcor GW and Cochran WG, Statistical methods. 7.ed. Iowa State University, Iowa, , 1980.

[20]El-Sayed, Y.S. and T.T. Saad, Subacute intoxication of a deltamethrin-based preparation (Butox) 5\% EC) in monosex Nile tilapia, Oreochromis niloticus L. Basic Clin Pharmacol Toxicol, 2008. 102(3): p. 293-9. http://dx.doi.org/10.1111/j.1742-7843.2007.00157.x.

[21] Sweilum, M.A., Effect of sublethal toxicity of some pesticides on growth parameters, hematological properties and total production of the Nile tilapia (Oreochromis niloticus) and water quality of ponds. . Aqua. Res., 2006. 37: p. 1079 - 1089. http://dx.doi.org/10.1111/j.13652109.2006.01531.x.

[22] Jyothi, B. and G. Narayan, Certain pesticide-induced carbohydrate metabolic disorders in the serum of freshwater fish Clarias batrachus (Linn.). Food Chem Toxicol, 1999. 37(4): p. 417-21. http://dx.doi.org/10.1016/S0278-6915(99)00020-4.

[23] Singh, S.K., et al., Toxicity of malathion and carbaryl pesticides: effects on some biochemical profiles of the freshwater fish Colisa fasciatus. Bull Environ Contam Toxicol, 2004. 72(3): p. 592-9. http://dx.doi.org/10.1007/s00128-004-0285-4.

[24] Matos, P., et al., Biochemical and histological hepatic changes of Nile tilapia Oreochromis niloticus exposed to carbaryl. Pesticide Biochemistry and Physiology, 2007. 89(1): p. 73-80. http://dx.doi.org/10.1016/j.pestbp.2007.03.002.

[25]Dutta, H.M., et al., Malathion induced changes in the serum proteins and hematological parameters of an Indian catfish Heteropneustes fossilis (Bloch). Bull Environ Contam Toxicol, 1992. 49(1): p. 91-7. http://dx.doi.org/10.1007/BF00193346.

[26]Fouda, F.M., Hematological and biochemical studies on the effects of biological and a chemical pesticides on the Nile catfish, Clarias gariepinus. J. Egypt. Ger. Soc. Zool., 2004. 43: p. 77 - 97.

[27] Oruc, E.O. and D. Usta, Evaluation of oxidative stress responses and neurotoxicity potential of diazinon in different tissues of Cyprinus carpio. Environ Toxicol Pharmacol, 2007. 23(1): p. 48-55. http://dx.doi.org/10.1016/j.etap.2006.06.005.

[28]El-Amin, A.I.M., Physiological effects of pesticides on common carp, Cyprinus carpio. . M.Sc. Thesis, Fac. of Sci. Cairo Univ., Egypt., 2002.

[29] Sharaf - El-Deen, K. and N.A. Abdel-Hamid, Sublethal effects of copper sulfate malathion and paraquat on protein pattern of Oreochromis niloticus. Egypt. J. Aquat. Biol. \& Fish. 2002. 6: p. 167 - 182.

[30]El-Elaimy, I.A., et al., Toxicological effects of dimethoate on proteins and nucleic acids metabolism in tissues of fresh water fish, Sarotherodon galilaeus. . J. Egypt. Ger. Soc. Zool., 1994. 14: p. 473 - 494.

[31]Quabius, E.S., et al., The influence of polychlorinated biphenyl 126 on tilapia (Oreochromis mossambicus) liver. Comparative Biochemistry and Physiology Part A: Molecular \& Integrative Physiology, 1998. 120(1): p. 57-63. http://dx.doi.org/10.1016/S1095-6433(98)10010-7.

[32] Fischer-Scherl, T., et al., Morphological effects of acute and chronic atrazine exposure in rainbow trout (Oncorhynchus mykiss). Arch Environ Contam Toxicol, 1991. 20(4): p. 454-61. http://dx.doi.org/10.1007/BF01065833.

[33] Sharma, B., Effect of carbaryl on some biochemical constituents of the blood and liver of Clarias batrachus, a fresh-water teleost. J Toxicol Sci, 1999. 24(3): p. 157-64. http://dx.doi.org/10.2131/jts.24.3 157.

[34]Fayed, H.M., et al., Biological responses of the Nile Tilapia, Oreochromis niloticus and the Nile catfish, Clarias gariepinus induced by agricultural and industrial pollutants. Union of Arab Biologists, 2001. 16: p. 543-568.

[35] Mekkawy, A.A., et al., Comparative studies on the effects of herbicide atrazine on some blood constituents and protein electrophoretic patterns of Oreochromis niloticus and Chrysichthyes auratus at Assiut, Egypt. . J. Egypt. Ger.Soc. Zool, , 1996. 19: p. 283 - 319.

[36] Guyton, A., Text Book of Medical physiology 5th Ed. Sanders Co; Philadelphia, , 1976: p. 668-686.

[37] Ceron, J., et al., Metabolic effects of diazinon on the European eel, Anguilla anguilla. J. Environ. Sc. Health. Part B (Pesticides Food Contaminants and Agri. Wastes), 1996. 31: p. 1029-1040.

[38]Fouda, F.M. and M.A. Azab, A comparative toxicological study on the effects of a biological and a chemical pesticides on the liver of Nile catfish, Clarias gariepinus. . J.Egypt. Ger. Soc. Zool., 2003. 40: p. 105-120.

[39]El-Ezabi, M.M., et al., Effects of some toxicants on the freshwater fish, Oreochromis niloticus. . J. Egypt. Ger. Soc. Zool., 2001.36 : p. 470 - 434.

[40] Abd-El-Razek, E., Effect of herbicide ametryne on some biological aspects of the common carp. . M.Sc. Thesis, Faculty of Science, Zagazig University, Egypt., 1994.

[41] Rao, P.P., K.V. Joseph, and K.J. Rao, Histopathological and biochemical changes in the liver of a freshwater fish exposed to heptachlor. J. Nat. Conserv., 1990. 2: p. 133 - 137. 